\title{
CD40 Monoclonal Antibody and OK432 \\ Synergistically Promote the Activation of Dendritic Cells in Immunotherapy
}

Juan Zhang

Shenzhen University

Lei Wang

Mengchao Hepatobiliary Hospital of Fujian Medical University

Shuyi Li

Shenzhen University General Hospital

\section{Xuefeng Gao}

Shenzhen University General Hospital

Zhong Liu ( $\square$ liuzhong5979@szu.edu.cn )

Shenzhen University General Hospital https://orcid.org/0000-0003-0961-276X

\section{Research Article}

Keywords: Colorectal cancer, pulmonary metastasis, cytotoxic T lymphocytes, CD40 mAb, OK-432

Posted Date: February 17th, 2022

DOI: https://doi.org/10.21203/rs.3.rs-1113492/v1

License: (9) This work is licensed under a Creative Commons Attribution 4.0 International License. Read Full License 


\section{Abstract}

Background: Colorectal cancer (CRC) with pulmonary-metastasis usually indicates a poor prognosis, whereas patients may benefit from adoptive cell therapy. Tumor specific cytotoxic T lymphocytes (CTLs) have been reported to be a promising treatment for CRC. However, the antitumor effect of CTLs remains limited, partially due to insufficient production of effector cells via the activation with antigen-presenting dendritic cells (DCs).

Method: Here we presented that a combination of CD40 mAb and Picibanil (OK-432) could significantly enhance the activation of CTLs by DCs, both in vitro and in vivo. Flow cytometric, colon cancer mouse model, and pathological staining were employed to demonstrate the specific functions.

Results: This approach promoted the maturation of DCs, augmented the production of stimulatory cytokines, and suppressed the secretion of inhibitory cytokines. Additionally, it facilitated the killing efficiency of CTLs via stimulating their proliferation while restrained the number of Tregs, concomitantly with the positive regulation of corresponding cytokines. Furthermore, the combined unit could hurdle the expansion of tumor cells on metastatic lung in colon cancer mouse model.

Conclusion: Collectively, the combination of CD40-mAb and OK-432 can facilitate the maturation of DCs and enhance the cytotoxicity of $\mathrm{T}$ cells, which may be a promising approach for clinical treatment against CRC.

\section{Introduction}

Colorectal cancer (CRC), as a leading lethal malignancy, has been a major cause of cancer-associated death worldwide [1]. Despite of improved five-year survival via early detection, the metastasis to distant organ such as lung has been the prominent cause for mortality. Approximately $25 \%$ of CRC patients suffer from stage IV disease, and near 25 50\% patients with early stages will eventually progress to metastatic disease, which indicates poor prognosis and $12.5 \%$ of five-year survival $[2,3]$. Therefore, more effective approaches against metastatic CRC (mCRC) are on urgent demand.

Immunotherapy, designed to boost the natural defense of immune system, has powerfully altered the landscape of malignancy treatment, including mCRC. It has exhibited improved antitumor immunity and tremendous potential for reversing the immunosuppression induced by tumor. For instance, the immune checkpoint inhibitors (ICls) have lead the immunotherapy field to regain a major influence in tumor research [4]. However, the immune-related adverse events (irAEs) are inevitable, as ICls usually elicits long-lasting clinical responses via reactivating an exhausted immune response. Whereas, adoptive cell therapy (ACT) may provide the cancer-targeting immune infiltrate and specific immune response [5]. It can keep long-term durable in solid tumor, showing a peculiar property, which was ever hardly achievable for chemotherapy $[1,6]$. Thereinto, DCs play a vital role in the immune response especially the stimulation of CTLs. Landmark studies have demonstrated that CD8+ CTL density are strongly associated with disease-free and overall survival in CRC patients, which has been employed as a prognostic biomarker for 
the survival of CRC patients [7, 8]. Adoptive cell therapy (ACT) has displayed powerful potential in the development of CRC treatment in the past decades. And CTL-mediated inflammatory response to tumor cells acts a critical role in suppressing the progression of carcinogenesis [9]. Therefore, approaches improving the generation of CTLs are vital for the immunotherapy of CRC patients.

The cytotoxicity of CTLs critically depend on dendritic cells (DCs) [10]. As professional antigen-presenting cells (APCs), DCs play essential roles in both innate and adaptive immunity system upon pathogen invasion [11]. Immature DCs take up antigens through endocytose phagocytosis, micro- or macropinocytosis, and endocytosis using Fc receptors, integrins, C-type lectin receptors, apoptotic cell receptors, and scavenger receptors. The mature DCs can transport cancer-associated antigens to the draining lymph node where T cell priming and activation can occur to generate the CTLs [12]. Various studies have shown that CTLs induced by DCs can enhance the antitumor effect against CRC [13-16]. Therefore, targeting DCs may confer a feasible strategy for CRC immunotherapy. The inhibitory cytokine (such as IL10 and TGF- $\beta$ ) and stimulatory cytokine (such as IL-12 and IFN- $\gamma$ ) function opposite roles in the process of CTL generation $[17,18]$. The former is involved in the suppression of T cells whereas the later stimulates them. Produced by Tregs, IL-10 and TGF- $\beta$ majorly function as a suppressive role in the immune system. As a pro-inflammatory cytokine, IL-12 contributes to the activation of T cell and NK cell, and the production of IFN- $y$ that favors the differentiation of Th1 cell. It can be produced by mature DCs upon pathogen infection [19]. Besides, IL-12 can suppress the proliferation of Tregs and the Foxp3 level, favoring the outgrowth of non-Tregs. Collectively, IL-12 and IFN-y majorly function as a stimulator favoring the activation of cytotoxic cells [19]. Furthermore, DCs themselves also act as direct killer targeting on tumor cells and induce the dying cells to release tumor-related antigens [20].

CD40, a cell-surface member of tumor necrosis factor (TNF) receptor superfamily, is expressed on variety of APCs. As a costimulatory molecule, it is vital for the activation of APCs and CD8+ T cells [21]. The CD40-CD40L signaling plays a central role in the regulation of the immune response through an interaction between T cells and APCs. They can help the antigen presentation and promote the expression of costimulatory molecules, which allows the maturation of DCs and stimulation of $\mathrm{T}$ cells. Upon activation of lymphocytes, the binding of CD40L on the surface of T cells with CD40 provides costimulatory molecules required to activate naive $T$ cells thereby amplifying the immune response. Preclinical investigations showed that CD40-activated DCs are poised to prime or activate tumor-specific T cells [22]. Moreover, CD40 activated macrophages were shown to infiltrate tumors and destroy tumor stroma via tumoricidal activity [23]. Besides, as a membrane antigen, CD40 can regulate the production of costimulatory cytokine IL-12. These data inspired the utilization of CD40 agonists, particularly the CD40 monoclonal antibodies (mAb), as a novel approach for cancer immunotherapy. It has shown a feasibility in both pre-clinical and clinical settings [21]. Evidences suggests that the activation of CD40 display critical role in the conversion of "cold tumor" to "hot tumor", which makes tumor more susceptible to immune checkpoint inhibitors [24]. In the past few years, CD40 mAb combined with other therapies have shown attractive efficacy, which is potentially important to extend the effective range of CD40 mAb. It has been reported that CD40 antibodies with varying formulations show tolerable and feasible function in patients with mesothelioma, pancreatic cancer, and others, when combined with chemotherapy [22], 
whereby the companion may codetermine the terminal outcomes, which needs more attention to be paid. Although CD40 mAb is potential for the immunotherapy, it is deficient as CD40 mAb not only stimulate the production of stimulatory cytokine IL-12, but also the inhibitory cytokine IL-10 [25]. Therefore, it is necessary to explore an adjuvant agent to neutralize the side effect of CD40 mAb.

OK-432 is a lyophilized preparation derived from penicillin-inactivated Streptococcus pyrogene [26]. It can stimulate the maturation of DCs thereby priming CTL and natural killer (NK) cell response against tumor cells. Because mature DCs can produce proinflammatory cytokines, which is beneficial to the proliferation of CTLs. $[27,28]$. And this cytotoxicity is mediated by the CD40/CD40 ligand axis, as CD40 ligand on DCs can interact with CD40 on tumor cells [27]. The effect of OK-432 on DCs may be achieved by following steps: 1). Induce the generation of IL-12 and IFN-ץ. 2). Upregulate the expression of CD40, CD80, CD86, adhesion molecules (ICAM-1), etc. 3). Stimulate the peptide-specific cytotoxic lymphocytes (CTLs). And these data endowed OK-432 with potential in anticancer immunotherapy, indicating a feasible clinical application, which has been approved by clinical observation in CRC $[29,30]$.

Given the above proofs, here we proposed that the combination of CD40 mAb and OK-432 may confer a synergistic effect on the activation of DCs, which determine the generation of CTLs. We employed a mouse model of CRC lung metastasis to demonstrate the therapeutic effect of this combination.

\section{Materials And Methods}

\section{Reagents and cell lines}

OK-432 was purchased from T\&L Biological Technology (GMP-TL107-0100). CD40 mAb was purchased from AdipoGen (AG-20B-0036PF). Antibodies used in the FCM were purchased from Miltenyi Biotec, which were listed in supplemental table $\mathrm{S} 1$. The information of cytokines used in the experiment can also be searched in Supplemental Table S1. Human colorectal cancer SW-620 cells and mouse colon cancer CT26 cells were obtained from the Institute of General Surgery, Chinese PLA General Hospital (Beijing, China). SW-620 and CT26 cells were cultured in Dulbecco's modified Eagle's medium (Gibco, 11965092), supplemented with 10\% heat inactivated fetal bovine serum (FBS, Gibco, 16000044), 100x PenicillinStreptomycin Solution (Corning ${ }^{\circledR}, 30-002-\mathrm{Cl}, \mathrm{USA}$ ) at $37^{\circ} \mathrm{C}$ in a $5 \% \mathrm{CO}_{2}$ incubator (Thermo Scientific, Waltham, MA, USA). When the cells were over $90 \%$ confluent, they were detached by $0.25 \%$ Trypsin (Gibco, 25200056) and passaged at a ratio of 1:4.

\section{Ethics statement}

The experimental mice used in this study were obtained from the Laboratory Animal Center of the Academy of Military Medical Science. All experimental and postoperative animal care procedures were performed according to the protocols approved by the Animal Care and Use Committee of the Chinese PLA General Hospital and the National Institute of Health's Guidelines for the Care and Use of Laboratory Animals. And the experiments were performed in the experimental animal room of Dalian Medical University. As the experimental performance is relatively harmless, we did not use anesthetic until the last 
step of sacrifice, which was terminated by $\mathrm{CO}_{2}$ narcosis, which was performed by trained personnel. According to the PACUC Guideline, mice were displaced in 10-liter volume chamber (less than five mice per cage), and compressed $\mathrm{CO}_{2}$ gas in cylinders were used at a flow rate 5 liter per minute $(50 \%$ of the chamber volume), lasting for 2-3 minutes until the mice were unconscious, and then cervical dislocation was performed.

The study on clinical samples was conducted according to the guidelines of the Declaration of Helsinki, and approved by the Ethics Committee of The First Affiliated Hospital of Dalian Medical University (Dalian, Liaoning, China). Written informed consent was obtained from all subjects in accordance with the Declaration of Helsinki.

\section{Preparation of mouse bone marrow DCs (BMDCs)}

The collection of BMDCs was performed according to the previous study [31]. Briefly, femur bones were isolated from six-week-old Balb/c mice. And the bone marrow suspension was collected by flushing the femurs, which will be rinsed and re-suspended at a final density of $10 \times 10^{6} \mathrm{cell} / \mathrm{s} / \mathrm{ml}$ for subsequent seeding. The cells were cultured in regular medium supplemented with $20 \mathrm{ng} / \mathrm{ml} \mathrm{rmGM-CSF}$ for six days, during which the medium with rmGM-CSF was refreshed every three days. And then the non-adherent cells Resuspend the pellet of cells and diluted them to achieve cell density of were harvested as BMDCs that will be analyzed by FACS.

\section{Flow cytometer}

The DCs were identified by CD11c-FITC antibody and analyzed by FACS. The subsets of DCs were sorted by corresponding antibodies such as CD8a, CD80, and CD86. The CD8 $a^{P 0 s}$ subset of DCs were purified by biotin-conjugated antibody cocktail. And the $\mathrm{CD} 8 \mathrm{a}^{\mathrm{Pos}}$ subset of DCs was then incubated with either vehicle control or test groups including positive control TNF-a, OK-432, CD40 mAb, and combination of OK-432 and CD40 mAb.

The proportions of regarding subtypes in T cells were detected by FCM. Briefly, lymphocytes were first incubated with regarding antibodies for 30 minutes at room temperature according to the manufacturer's instructions. For detection of Foxp3, cells were subjected to membrane rupture and stained with Foxp3-PE (Miltenti Biotec-3G3, Cologne, Germany) according to the manufacturer's protocol. All samples were examined by a FACS Calibur instrument (Becton Dickinson, USA) and the data were analyzed with FlowJo7.6.1 software. The level of interferon-gamma (IFN- $\gamma$ ) in the cell culture supernatant was determined using an enzyme-linked immunosorbent assay kit (Sigma- Aldrich, St. Louis, MO, USA). The in vitro cytotoxicity of the CTLs (effect cell, E) to the CT26 cells (target cell, T) was assessed using an LDH release assay kit.

\section{Preparation of CTLs}

The collection of splenic T cells was performed according to the previous study [32]. Briefly, mouse spleen was mashed until it was torn into fine parts. Filter and collect the cell suspension, which was then 
resuspended with RBC (red blood cell, Chinese Academy of Medical Sciences, Beijing, China) lysis buffer, to acquire the mononuclear cells. And the splenic cells were then purified by non-T cell depletion antibody cocktail. And the purity of T lymphocytes was checked by flow cytometry, using antibodies specific for purified T cells. And the collection of T cells derived from peripheral blood was performed as mentioned above, starting at the stage of red blood cell lysis.

Conventional CTLs and autologous CTLs were prepared in our laboratory as previously reported [33, 34]. Briefly, mDCs were co-cultured with T cells at a ratio of $1: 10$, supplemented with $100 \mathrm{ng} / \mathrm{mL} C D 3 \varepsilon \mathrm{mAb}$ and $10 \mathrm{ng} / \mathrm{mL} \mathrm{rm}-\mathrm{IL}-2$. The medium with IL-2 was refreshed every other day. And on the seventh day, CTLs can be harvested for the subsequent experiments.

\section{Animal models and in vivo experiments}

Six-week-old Balb/c female mice were purchased from the Beijing Experimental Animal Center of the Academy of Military Medical Sciences (Beijing, China). The colon cancer lung metastasis model was conducted according to the previous description [33]. Briefly, $1 \times 10^{5}$ mouse CT26 cells in $100 \mu \mathrm{L}$ phosphate-buffered saline were injected into the mice via the tail vein. On the fourth day, the tumorbearing mice were randomly divided into 7 groups with 4 mice each, including one vehicle and six treatment groups. Group 1, each mouse was treated with $200 \mu \mathrm{l}$ saline per day, marked as vehicle group; Group 2, mouse was treated with CTLs $\left(1 \times 10^{7}\right.$ cells $)$ induced by DC, marked as DC-CTL group; Group 3, mouse was treated with CTLs $\left(1 \times 10^{7}\right.$ cells $)$ induced by DC with anergic antigen, marked as Ag-DC-CTL group; and by the analogy, group 4 to group 7 was correspondingly marked. The treatments were applied for two weeks. At the end of two weeks, mice from each group were sacrificed to assess the antitumor effect.

\section{Counting of metastatic lung nodules}

Counting metastatic lung nodules and enrichment of lymphocytes from lung tissue were performed as previously described [33]. Briefly, nodule diameters of less than $0.5 \mathrm{~mm}, 0.5-1 \mathrm{~mm}, 1-2 \mathrm{~mm}$, and greater than $2 \mathrm{~mm}$ were classified as grade I, II, III, and IV metastasis, respectively. The total numbers of metastases were calculated according to the following formula: total metastasis number $=$ (grade I metastasis number $)+($ grade II metastasis number $\times 2)+($ grade III metastasis number $\times 3)+($ grade IV metastasis number $\times 4$ ). The left lung was digested and then the mononuclear cell suspensions were collected using discontinuous density gradient centrifugation with mouse lymphocyte separation medium (MP Biomedicals, Santa Ana, CA, USA).

\section{Cytotoxicity assay}

The in vitro cytotoxicity of CTLs to SW-620 cells was performed by an LDH release assay (Sigma-Aldrich, St. Louis, MO, USA), as described previously [35]. The target SW-620 cells were mixed with CTLs derived from different treatments (vehicle control, TNF-a, CD40, OK-432, and the combined group of CD40 and OK-432) 
at a ratio of 5:1 or 10:1 and were cultured for $24 \mathrm{~h}$. The optical density (OD) value was detected with a microplate reader at a wavelength of $492 \mathrm{~nm}$. The killing rate was calculated as follows: Cytotoxicity \%= [OD (Experimental) - OD (Effector spontaneous) - OD (Target spontaneous)] ×100 / [OD (Target maximum) - OD (Target spontaneous)].

\section{Haematoxylin Eosin (H\&E) staining}

The lungs of control and test groups were fixed with fresh $4 \%$ formalin for 24 hours at room temperature, followed by dehydration, transparent, and paraffin embedding. And then the samples were sliced into 4 $\mu \mathrm{m}$ sections. Then the tissue slides were stained by HE (Biosharp, BL700A) and the histological images were observed and photographed by Light Microscopy.

\section{Statistical analysis}

SPSS 21.0 statistical software was used for statistical analysis of the relevant data. Data are expressed as the mean \pm standard deviation. Differences between two groups were compared using T-tests. Differences among several groups were analyzed by one-way analysis of variance. $P<0.05$ was considered statistically significant. The survival rate was analyzed using the Kaplan-Meier method.

\section{Result}

\section{CD40 mAb and OK-432 show synergistical function in activation of human DCs}

First, we accessed the activation effect of CD40-mAb and OK-432 combination on human PBMC in vitro with three control groups (Neg-Ctrl: without any agent; Ag-Ctrl: $10 \mu \mathrm{g} / \mathrm{ml}$ anergic antigen; TNF-a: positive control with a concentration of $10 \mathrm{ng} / \mathrm{ml}$ ), and three test groups (CD40 mAb: $1 \mu \mathrm{g} / \mathrm{ml} ; 0 K-432: 10 \mu \mathrm{g} / \mathrm{ml}$, and 40432 group: $1 \mu \mathrm{g} / \mathrm{ml} \mathrm{CD} 40 \mathrm{mAb}$ plus $10 \mu \mathrm{g} / \mathrm{ml}$ OK-432). Subjected to MHC restriction, we firstly verified that the agent-treated groups showed a uniformity with the control groups (Fig. 1A). We measured the expression of CD83 to identify the maturity of human DCs [36], and found that expression of CD83 were increased by both CD40 Ab and OK-432, and their combination further enhanced the CD83 expression (Fig. 1B). Additionally, an increased ratio of type 1 DCs was detected (Fig. 1C), suggesting an enhanced potential of inducing the generation of CTLs [37]. More interestingly, the combination of CD 40 $\mathrm{Ab}$ and $\mathrm{OK}-432$ upregulated the production of IL-12, which was not detected in the single agent test group (Fig. 1D). IL-12 plays a critical role in both innate and adaptive antitumor immunity. IL-12 production by DCs can augment Th1 response and stimulate the differentiation and lytic capacity of CTL [38].

DCs induced by CD40 Ab and OK-432 benefit to promote the proliferation of cytotoxic $T$ cells while suppress Treg cells

Given the positive role of CD40 Ab and OK-432 in human DCs activation, we next investigated the potential effect of DCs on generating CTLs. We observed that human DCs induced by CD40 Ab or OK-432 could induce the generation of cytotoxic T cells (Fig. 2A). And the combination of CD40 Ab with OK-432 
could generate more $\mathrm{CD} 8^{+}$cells than their independent manner. Moreover, a higher level of IFN- $\gamma$ were produced in the combination manner (Fig. 2B), suggesting a stronger anti-tumor potential.

Meanwhile, we also found that the combination of CD40 Ab and OK-432 reduced the ratio of CD3+CD4+ T cells versus total T cells (Fig. $2 \mathrm{C}$ ), which otherwise indicates the increasement of CTLs. Treg cells are opposite to the stimulation of T cells. Our data showed that the proliferation of Treg cells originating from peripheral blood are suppressed by CD40 Ab and OK-432, especially in the combination group (Fig. 2D) which can be verified by the expression of Foxp3 (Fig. 2E), a specific marker of Tregs.

Collectively, these results demonstrated that the combination of CD $40 \mathrm{Ab}$ and OK-432 not only boosts the maturation of mouse DCs, but also human DCs. Both are feasible to the stimulation of CTLs, providing a preclinical potential against the colorectal cancer.

\section{$\mathrm{CD} 40 \mathrm{Ab}$ and $\mathrm{OK}-432$ show synergistical function in activation of DCs}

DCs were defined by expression of co-stimulatory signals CD80 and CD86 (ligands of CD28 expressed on $T$ cells). We identified the $C D 8 a^{+} C D 11 c^{+} C D 11 b^{-}$subset of DCs (Supplementary Fig.S1), which is committed to play a critical role in $\mathrm{CD}^{+} \mathrm{T}$ cell activation $[39,40]$. The cytokines and corresponding agents were detected to assess the immune response to CD40 mAb and OK-432 combination (termed as 40-432).

The combination of CD40 mAb or OK-432 showed a stronger effect on stimulating the expression CD80 (Fig. 3A) and CD86 (Fig. 3B) compared to the single agent groups. Besides, 40-432 significantly stimulated the generation of $C D 11 c^{+} C D 11 b^{-} C D 8^{+}$subset of $D C s$, a subset specially contributes to the stimulation of $\mathrm{CD}^{+} \mathrm{T}$ cells, which was superior to the other agents (Fig. $3 \mathrm{C}$ ). The stimulatory cytokine IL12 plays a critical role in both innate and adaptive antitumor immunity [38]. In contrast, the level of inhibitory cytokine IL-10 was not significantly changed after stimulation with OK-432. The combined 40432 was found to upregulate IL-12 without affecting the level of IL-10. Apart from, the combined group 40-432 as well modestly affect the secretion of TGF- $\beta$, which is better than OK-432 group (Fig. 3D), indicating the advantage of combination.

\section{DCs induced by CD40 mAb and OK-432 confer T cells with enhanced killing efficiency}

Given the positive role of CD40 mAb and OK-432 in DCs activation, we next investigated the potential effect of DCs on cytotoxic T cells. We observed that CD40 mAb and OK-432, alone or in combination manner, could reduce the ratio of $\mathrm{CD}^{+} \mathrm{CD}^{+} \mathrm{T}$ helper cells (Fig. 4A), indicating a reciprocal relationship between $\mathrm{CD} 4^{+}$and $\mathrm{CD} 8^{+}$cells. And we found that the combined group restrains the generation of inhibitory cell Tregs $\left(\mathrm{CD} 4^{+} \mathrm{CD} 25^{+} \mathrm{Foxp} 3^{+}\right)$, indicating a superior role of combined group to the other ones 
(Fig. 4B). Functionally, the production of IFN-y derived from T cells was increased when treated with CD40 $\mathrm{mAb}$ or OK-432, whereas even higher level of IFN- $\gamma$ was detected in the combination group (Fig. 4C).

We then tested the cytotoxicity of CTLs induced by CD40 mAb and OK-432 on CT26 CRC cell line. At effector: target (E:T) ratio of 5:1, CTLs induced by the combination of CD40 mAb and OK-432 showed a stronger cytotoxicity against CT26 cells than any single agents (Fig. 4D). And the killing efficacy was twofold enhanced at effector: target (E:T) ratio of 10:1.

CTLs induced by CD40 mAb and OK-432 show potent feasibility in the treatment of colon cancer model with pulmonary-metastasis

Based on the achievement of functional CTLs, we next test the possible role of CTLs in the treatment of CRC mouse model with pulmonary-metastasis. Mice involved in this experiment were divided into seven groups. Each group were administered with corresponding CTLs originating from pertinent DCs. Briefly, mice were administered with $1 * 10^{7}$ CTLs twice a week via tail vein injection? which were maintained for four courses. The morphology and weight of the mouse lungs were accessed to compare the tumor suppression and metastasis control efficiency of CTLs induced by different agents. We observed that the combination of CD40 mAb with OK-432 outperformed single agent in restraining the pulmonarymetastasis (Fig. 5A). Both the ratio of lung weight/body weight (Fig. 5B) and the number of pulmonary metastatic nodes (Fig. 5D) delineated the most in the group of 40-432 group. Furthermore, H\&E staining of the lung indicated fewer neoplastic cells in the 40-432 group compared to the others (Fig. 5C). Thus, CTLs generated by CD40 mAb-OK-432 induced DCs had a better anti-tumor effect in controlling CRC lung metastasis.

DCs induced by CD40 mAb and OK-432 contribute to the proliferation of CTLs while facilitate the suppression of Tregs in vivo

Ensured by the phenotype in vivo that combination of CD 40 mAb and OK-432 was beneficial to CRC model with pulmonary-metastasis, we next investigated where can the CTLs function. The cell sources were derived from three types of tissues: peripheral blood, spleen, and lung. When treated with the four agent-treated groups, the number of $\mathrm{CD}^{+} \mathrm{T}$ cells increase with varying degrees of proliferation. And the combined group shows a significant enhancement (Fig. 6A). In addition, the proliferation of Tregs originating from the three sources are all suppressed by the four agent-treated groups, whereas the combinational group shows a prominent suppression (Fig. 6B).

Furtherly, the cytokines tightly involved in the stimulation of CTLs were also detected in peripheral blood. We observed that the production of inhibitory cytokines IL-10 and TGF- $\beta$ are both restrained by the agenttreated groups, and the combinational group contributes the significant change (Fig. 7A, B). Meanwhile, the stimulatory cytokines IL-12 and IFN- $y$ were also detected. Results demonstrated that the four agenttreated groups facilitate the enhancement of stimulatory cytokine IL-12 and IFN- - , whereas the combined group exhibits the outstanding function (Fig. 7C, D). 


\section{Discussion}

Development with the advanced cytotoxic agents and molecular-targeted treatment, the clinical outcomes of various cancers have been improved. However, the ratio of overall survival is still dismal including colon cancer, as patients usually suffer from advanced cancers such as colon cancer with pulmonarymetastasis, which have not been virtually improved [41-43]. Therefore, new approaches against such cancers are desired. And immunotherapy displays a prominent potential. As an antigen-presenting cell, DC acts as a vital component in immune system. It can present the tumor-associated antigens to cytotoxic cells, which release cytokines targeting tumor cells. Additionally, DCs also control the immune tolerance and immunity $[10,12]$. Thus, target on DCs confers on feasible strategy for cancer immunotherapy.

The immune components in tumor microenvironment (TME) are crucial for the immunotherapy [44]. Regulatory T cells (Tregs), display a vital role in suppressing the activity of CTLs, conferring to immune escaping and the treatment failure. And the increasing number of infiltrated Tregs generally indicates a poor prognosis $[45,46]$. Among the $C D 4^{+} \mathrm{T}$ cells, there are approximately $5 \%-10 \%$ Tregs, which secret cytokines interleukin-10 (IL-10) and transforming growth factor $\beta$ (TGF $\beta$ ) [47]. In normal, Tregs maintain the immune homeostasis and induce the immune tolerance. And abnormally, the dysregulation of Tregs is associated with the induction of immune tolerance, contributing to the escape of tumor cells from being attacked by immune cells. Mounting evidences have demonstrated that Treg-mediated immunosuppression is the major cause leading to the failure of tumor treatment [48, 49]. Oppositely, CTLs act as a contrary role with Tregs. They secret IFN- $y$ that facilitates the killing of invasion. CD80 and CD86 function in dendritic cells (DCs) licensing and CTLs activating. DC presents the complex of major histocompatibility complex class II (MHC class II)-peptide, which can interact with receptor on T helper cell (Th). Upregulation of CD80 and CD86 license the DCs to interact with CD8 ${ }^{+} T$ cells $[10,50]$. Therefore, as a precondition, activation of DCs is indispensable for the stimulation of CTLs. The enhancement of DC activation certainly facilitates the generation of CTLs. One of the feasible approaches is to utilize the CD40 agonists, which can activate the DCs and promote the secretion of IL-12, furthermore turn the "cold" tumor to "hot" one [24]. However, the single-agent antitumor activity of CD40 agonists was not enough. And they usually combined with other adjuvants.

Clued by the previous studies, we found that OK-432 as an adjuvant is beneficial to the treatment of colon cancer via the CD40/CD40L axis [22,27]. Then, the following question is whether the combination of CD40 agonists and OK-432 work with a synergistic function? And we employed the CD40 monoclonal antibody to elevate such speculation, which has been demonstrated by the given data. We observed that the combined group (CD40 mAb and OK-432) endows DCs with stronger potential to stimulate T cells, and prominent cytotoxic to tumor cells. Besides, the combination facilitates the expression of the costimulatory signals (CD80 and CD86) involved in the activation of DCs and the production of stimulatory cytokines (IL-12 and IFN- $\gamma$ ) involved in the stimulation of T cells. Meanwhile, it also suppresses the generation of Tregs and inhibitory cytokines (IL-10 and TGF- $\beta$ ). Collectively, the combination of CD40 $\mathrm{mAb}$ and $\mathrm{OK}-432$ provides a promising strategy to facilitate the immunotherapy. 


\section{Conclusion}

Collectively, we demonstrated that the combination of CD40-mAb and OK-432 can promoted the maturation of DCs, augmented the production of stimulatory cytokines, and suppressed the secretion of inhibitory cytokines, which then significantly enhance the activation of CTLs by DCs, both in vitro and in vivo. The combination of CD40-mAb and OK-432 may provide a promising approach for clinical treatment against CRC.

\section{Declarations}

\section{Ethics approval and consent to participate}

The experimental mice used in this study were obtained from the Laboratory Animal Center of the Academy of Military Medical Science. All experimental and postoperative animal care procedures were performed according to the protocols approved by the Animal Care and Use Committee of the Chinese PLA General Hospital and the National Institute of Health's Guidelines for the Care and Use of Laboratory Animals.

The study on clinical samples was conducted according to the guidelines of the Declaration of Helsinki, and approved by the Ethics Committee of The First Affiliated Hospital of Dalian Medical University (Dalian, Liaoning, China). Written informed consent was obtained from all subjects in accordance with the Declaration of Helsinki.

\section{Consent for publication}

Not applicable.

\section{Availability of data and materials}

All data generated or analysed during this study are included in this published article.

\section{Competing interests}

The authors declare no potential conflict of financial interest.

\section{Funding}

This work was supported by grants Natural Science Foundation of Shenzhen University General Hospital (SUGH2018QD050), Shenzhen Key Laboratory of Precision Medicine for Hematological Malignancies, and Sanming Project of Medicine in Shenzhen (SZSM202111004).

Author's contributions 
L.W designed and performed the experiments, analyzed the data, and modified the manuscript. J.Z wrote the manuscript. X.G. modified the manuscript. L.S. provided technical help. Z.L. designed the experiments, supervised the study, reviewed, and approved the manuscript.

\section{Acknowledgments}

Thanks to the grants mentioned above supporting the expense of the whole work. We also thank the colleagues of Liu's lab proposing valuable advices on experimental design and performance.

\section{References}

1. Ganesh K, et al. Immunotherapy in colorectal cancer: rationale, challenges and potential. Nat Rev Gastroenterol Hepatol. 2019;16(6):361-75.

2. Dai Y, et al. Perspectives on Immunotherapy of Metastatic Colorectal Cancer. Front Oncol. 2021;11:659964.

3. Bounaix Morand du Puch. C., et al., Chemotherapy outcome predictive effectiveness by the Oncogramme: pilot trial on stage-IV colorectal cancer. J Transl Med. 2016;14:10.

4. Robert C. A decade of immune-checkpoint inhibitors in cancer therapy. Nat Commun. 2020;11(1):3801.

5. Grosser R, et al. Combination Immunotherapy with CAR T Cells and Checkpoint Blockade for the Treatment of Solid Tumors. Cancer Cell. 2019;36(5):471-82.

6. Sumransub N, et al. Advances and new frontiers for immunotherapy in colorectal cancer: Setting the stage for neoadjuvant success? Mol Ther Oncolytics. 2021;22:1-12.

7. Galon J. Type, Density, and Location of Immune Cells Within Human Colorectal Tumors Predict Clinical Outcome. Science. 2006;313(5795):1960-4.

8. Mei Z, et al. Tumour-infiltrating inflammation and prognosis in colorectal cancer: systematic review and meta-analysis. Br J Cancer. 2014;110(6):1595-605.

9. Prizment AE, et al. Cytotoxic T Cells and Granzyme B Associated with Improved Colorectal Cancer Survival in a Prospective Cohort of Older Women. Cancer Epidemiol Biomarkers Prev. 2017;26(4):622-31.

10. Wculek SK, et al. Dendritic cells in cancer immunology and immunotherapy. Nat Rev Immunol. 2020;20(1):7-24.

11. Bol KF, et al. Dendritic Cell-Based Immunotherapy: State of the Art and Beyond. Clin Cancer Res. 2016;22(8):1897-906.

12. Qian C, Cao X. Dendritic cells in the regulation of immunity and inflammation. Semin Immunol. 2018;35:3-11.

13. Xing $X$, et al. Enhanced antitumor effect of cytotoxic $T$ lymphocytes induced by dendritic cells pulsed with colorectal cancer cell lysate expressing alpha-Gal epitopes. Oncol Lett. 2019;18(1):864-71. 
14. Guan Y, et al. FOLFOX Chemotherapy Ameliorates CD8 T Lymphocyte Exhaustion and Enhances Checkpoint Blockade Efficacy in Colorectal Cancer. Front Oncol. 2020;10:586.

15. Calik I, et al., Intratumoral Cytotoxic T-Lymphocyte Density and PD-L 1 Expression Are Prognostic Biomarkers for Patients with Colorectal Cancer. Medicina (Kaunas), 2019. 55(11).

16. Idos GE, et al. The Prognostic Implications of Tumor Infiltrating Lymphocytes in Colorectal Cancer: A Systematic Review and Meta-Analysis. Sci Rep. 2020;10(1):3360.

17. Anderson DA 3rd, Murphy KM, Briseno CG. Development, Diversity, and Function of Dendritic Cells in Mouse and Human. Cold Spring Harb Perspect Biol, 2018. 10(11).

18. Sathe P, Wu L. The network of cytokines, receptors and transcription factors governing the development of dendritic cell subsets. Protein Cell. 2011;2(8):620-30.

19. Trinchieri G. Interleukin-12 and the regulation of innate resistance and adaptive immunity. Nat Rev Immunol. 2003;3(2):133-46.

20. Sozzani S, Prete AD, Bosisio D. Dendritic cell recruitment and activation in autoimmunity. J Autoimmun. 2017;85:126-40.

21. Djureinovic D, Wang M, Kluger HM. Agonistic CD40 Antibodies in Cancer Treatment. Cancers (Basel), 2021. 13(6).

22. Karnell JL, et al. Targeting the CD40-CD40L pathway in autoimmune diseases: Humoral immunity and beyond. Adv Drug Deliv Rev. 2019;141:92-103.

23. Beatty $\mathrm{GL}$, et al. CD40 agonists alter tumor stroma and show efficacy against pancreatic carcinoma in mice and humans. Science. 2011;331(6024):1612-6.

24. Vonderheide RH. CD40 Agonist Antibodies in Cancer Immunotherapy. Annu Rev Med. 2020;71:4758.

25. Dawicki W, et al. CD40 signaling augments IL-10 expression and the tolerogenicity of IL-10-induced regulatory dendritic cells. PLoS ONE. 2021;16(4):e0248290.

26. Ryoma Y, et al. Biological effect of OK-432 (picibanil) and possible application to dendritic cell therapy. Anticancer Res. 2004;24(5C):3295-301.

27. Hill KS, et al. OK432-activated human dendritic cells kill tumor cells via CD40/CD40 ligand interactions. J Immunol. 2008;181(5):3108-15.

28. Oba MS, et al. The efficacy of adjuvant immunochemotherapy with OK-432 after curative resection of gastric cancer: an individual patient data meta-analysis of randomized controlled trials. Gastric Cancer. 2016;19(2):616-24.

29. Nio Y, et al. Orally administered streptococcal preparation, OK-432 augments the antitumor immunity of patients with gastric or colorectal cancer. Biotherapy. 1990;2(3):213-22.

30. Nakagawa $\mathrm{H}$, et al. In vivo immunological antitumor effect of OK-432-stimulated dendritic cell transfer after radiofrequency ablation. Cancer Immunol Immunother. 2014;63(4):347-56.

31. Madaan A, et al., A stepwise procedure for isolation of murine bone marrow and generation of dendritic cells. Journal of Biological Methods, 2014. 1(1). 
32. Lim JF, Berger H, Su IH. Isolation and Activation of Murine Lymphocytes. J Vis Exp, 2016(116).

33. Wang $L$, et al. Arsenic trioxide inhibits lung metastasis of mouse colon cancer via reducing the infiltration of regulatory T cells. Tumour Biol. 2016;37(11):15165-73.

34. Yan Y, et al. Combined therapy with CTL cells and oncolytic adenovirus expressing IL-15-induced enhanced antitumor activity. Tumour Biol. 2015;36(6):4535-43.

35. Zhao XY, et al. Resveratrol and arsenic trioxide act synergistically to kill tumor cells in vitro and in vivo. PLoS ONE. 2014;9(6):e98925.

36. Li Z, et al. CD83: Activation Marker for Antigen Presenting Cells and Its Therapeutic Potential. Front Immunol. 2019;10:1312.

37. Cancel JC, et al. Are Conventional Type 1 Dendritic Cells Critical for Protective Antitumor Immunity and How? Front Immunol. 2019;10:9.

38. Mirlekar B, Pylayeva-Gupta Y. IL-12 Family Cytokines in Cancer and Immunotherapy. Cancers (Basel), 2021. 13(2).

39. Yadav PK, et al. Reciprocal changes in CD11c(+)CD11b(+) and CD11c(+)CD8alpha(+) dendritic cell subsets determine protective or permissive immune response in murine experimental VL. Vaccine. 2020;38(2):355-65.

40. Wu J, et al. Critical role of integrin CD11c in splenic dendritic cell capture of missing-self CD47 cells to induce adaptive immunity. Proc Natl Acad Sci U S A. 2018;115(26):6786-91.

41. Dekker E, et al. Colorectal cancer. The Lancet. 2019;394(10207):1467-80.

42. El Sissy C, et al., Therapeutic Implications of the Immunoscore in Patients with Colorectal Cancer. Cancers (Basel), 2021. 13(6).

43. Angell HK, et al. The Immunoscore: Colon Cancer and Beyond. Clin Cancer Res. 2020;26(2):332-9.

44. Bader JE, Voss K, Rathmell JC. Targeting Metabolism to Improve the Tumor Microenvironment for Cancer Immunotherapy. Mol Cell. 2020;78(6):1019-33.

45. Wang H, Franco F, Ho PC. Metabolic Regulation of Tregs in Cancer: Opportunities for Immunotherapy. Trends Cancer. 2017;3(8):583-92.

46. Bauer CA, et al. Dynamic Treg interactions with intratumoral APCs promote local CTL dysfunction. J Clin Invest. 2014;124(6):2425-40.

47. Thepmalee $C$, et al. Inhibition of IL-10 and TGF-beta receptors on dendritic cells enhances activation of effector T-cells to kill cholangiocarcinoma cells. Hum Vaccin Immunother. 2018;14(6):1423-31.

48. Yan S, Zhang Y, Sun B. The function and potential drug targets of tumour-associated Tregs for cancer immunotherapy. Sci China Life Sci. 2019;62(2):179-86.

49. Alissafi $T$, et al. Balancing cancer immunotherapy and immune-related adverse events: The emerging role of regulatory T cells. J Autoimmun. 2019;104:102310.

50. Farhood B, Najafi M, Mortezaee K. CD8(+) cytotoxic T lymphocytes in cancer immunotherapy: A review. J Cell Physiol. 2019;234(6):8509-21. 


\title{
Figures
}

\section{Figure 1}

\section{CD40 Ab and OK-432 show synergistical function in activation of human DCs.}

\begin{abstract}
A. Expression of HLA-DR in human DCs. The expression of HLA-DR shows a uniformity between the different types of DCs induced by regarding agents.
\end{abstract}

B. CD40 Ab and OK-432 synergistically activate the expression of CD83. The experiment was performed in six groups, including three control groups: Neg-Ctrl (without any agent), Ag-Ctrl (with anergic antigen), and TNF- $a$ (positive control), and three test groups: CD40 Ab, OK-432, and 40432 group (combinational group, CD40 Ab and OK-432). Compared to the control groups (Neg-Ctrl, Ag-Ctrl, and TNF-a), expression of CD83 can be separately improved by CD40 Ab, and OK-432, and the synergistic group 40432 was most significantly improved.

C. CD40 Ab and OK-432 synergistically improve the ratio of type $1 \mathrm{DCs}$ (DC1). The number of DC1 is separately increased by CD40 Ab, and OK-432, and the synergistic group 40432 was prominent.

D. Combination of CD40 Ab and OK-432 significantly promotes the production of IL-12. The secretion of inflammatory mediator IL-12 is strongly stimulated by combinational group (CD40 Ab+OK-432).

Value represents the mean \pm SEM. ${ }^{*} \mathrm{P}<0.05, * * \mathrm{P}<0.01$

\section{Figure 2}

DCs induced by CD40 Ab and OK-432 benefit to the proliferation of cytotoxic $\mathrm{T}$ cells while suppress the number of Tregs.

\begin{abstract}
A. DCs induced by CD40 Ab and OK-432 stimulate the generation of cytotoxic $T$ cells. $T$ cells derived from peripheral blood were treated with different types of DCs induced by relative agents. And the combinational group stimuli relatively more $\mathrm{CD} 8^{+}$cells.
\end{abstract}

B. DCs induced by CD40 Ab and OK-432 augment the secretion of IFN- - . The three agent-treated groups facilitate the production of stimulatory cytokine IFN- $\gamma$, and the combinational group displays an obvious advantage. 
C. Combination of $\mathrm{CD} 40 \mathrm{Ab}$ and $\mathrm{OK}-432$ reduces the number of $\mathrm{CD} 4^{+} \mathrm{T}$ cells. Compared to control groups, the three test groups reduced the ratio of $\mathrm{CD}^{+} \mathrm{CD} 4^{+} \mathrm{T}$ cells versus total $\mathrm{T}$ cells.

D. DCs induced by CD40 Ab and OK-432 restrain the production of Tregs. The proliferation of Tregs originating from peripheral blood are suppressed by the three test groups, especially the combinational group.

E. DCs induced by CD40 Ab and OK-432 suppress the expression of Foxp3. The three test groups obviously suppress the expression of Foxp3, and the combinational group is significant.

\section{Figure 3}

\section{CD40 Ab and OK-432 show synergistical function in activation of mouse DCs.}

A. CD40 Ab and OK-432 synergistically activate the expression of CD80. The experiment was performed in six groups, including three control groups and three test groups. Compared to the control groups (NegCtrl, Ag-Ctrl, and TNF-a), expression of CD80 can be separately improved by CD40 Ab, and OK-432, and the synergistic group was most significantly improved.

B. CD40 mAb and OK-432 synergistically activate the expression of CD86. The expression of CD86 showed the same tendency to CD80 in A.

C. CD40 mAb and OK-432 activate the population of $C D 11 c^{+} C D 11 b^{-} C D 8^{+}$subset of DCs. The synergistic group (CD40 Ab+OK-432) significantly improved the number of $C D 11 c^{+} C D 11 b^{-C D} 8^{+} D C s$.

D. Combination of CD40 Ab and OK-432 affects the secretion of TGF- $\beta$. Combination of CD40 Ab and OK-432 modestly affects the secretion of TGF- $\beta$.

Value represents the mean \pm SEM. ${ }^{*} P<0.05, * \star P<0.01$.

\section{Figure 4}

DCs induced by combination of CD40 Ab and OK-432 enhance the killing efficiency of T cells.

A. Combination of CD40 Ab and OK-432 reduces the number of CD4 ${ }^{+} \mathrm{T}$ cells. The experiment was designed as six groups, including three control groups: Neg-Ctrl (without any agent), Ag-Ctrl (with anergic antigen), and TNF-a (positive control), and three test groups: CD40 Ab, OK-432, and 40432 group (combinational group, CD40 Ab and OK-432). Cells were separately treated by the six group, and the 
number analysis of T cells was performed by FCM. Compared to control groups, the three test groups significantly reduced the ratio of $\mathrm{CD} 3^{+} \mathrm{CD} 4^{+} \mathrm{T}$ cells versus total $\mathrm{T}$ cells.

B. Combination of CD40 Ab and OK-432 reduces the number of Tregs. The test groups suppress the generation of inhibitory cell Tregs.

C. Combination of CD40 Ab and OK-432 promotes the secretion of IFN- $\gamma$. The test groups contribute to the production of IFN-y derived from T cells.

D. Combination of CD40 Ab and OK-432 displays high cytotoxic to CT26 cells. The cancer cells were treated with stimulated T cells at effector: target (E:T) ratios of 5:1 and 10:1. The higher ratio group shows higher killing efficiency. And in general, the test groups show stronger cytotoxicity.

\section{Figure 5}

\section{CTLs induced by CD40 Ab and OK-432 augment the killing efficiency of tumors on lungs in colon cancer model with pulmonary-metastasis.}
A. Lungs derived from mouse models treated with different types of CTLs. Mice involved in this experiment were divided into seven groups. All of CTLs administered to colon cancer models with pulmonary-metastasis were induced by corresponding DCs. And they can suppress the expansion of lung in the mouse model. And DCs activated by CD40 Ab and OK-432 elicit the most efficient CTLs, which furtherly restrain the expansion of lung compared to the separate agent group.
B. Statistics of lung to body weight ratio $(\mathrm{mg} / \mathrm{g})$ corresponding to $A$.
C. H\&E staining of lung slides. H\&E staining was employed to observe the lung morphology. The combinational group show the least tumor cells, compared to the others.
D. Statistics of tumor nodes corresponding to C.

Figure 6

DCs induced by CD40 Ab and OK-432 activate the proliferation of cytotoxic T cells while suppress the number of Tregs. 
A. DCs induced by CD40 Ab and OK-432 stimulate the generation of cytotoxic $\mathrm{T}$ cells. T cells derived from three different sources (peripheral blood, spleen, and lung) were separately treated with different types of DCs. Compared to the control groups, the four agent-treated groups show an increasing number of $\mathrm{CD}^{+}$cells, and the combinational group displays a significant proliferation.

B. DCs induced by CD40 Ab and OK-432 restrain the production of Tregs. The proliferation of Tregs originating from the three sources are all suppressed by the four agent-treated groups, especially the combinational group. 
Fig. 7
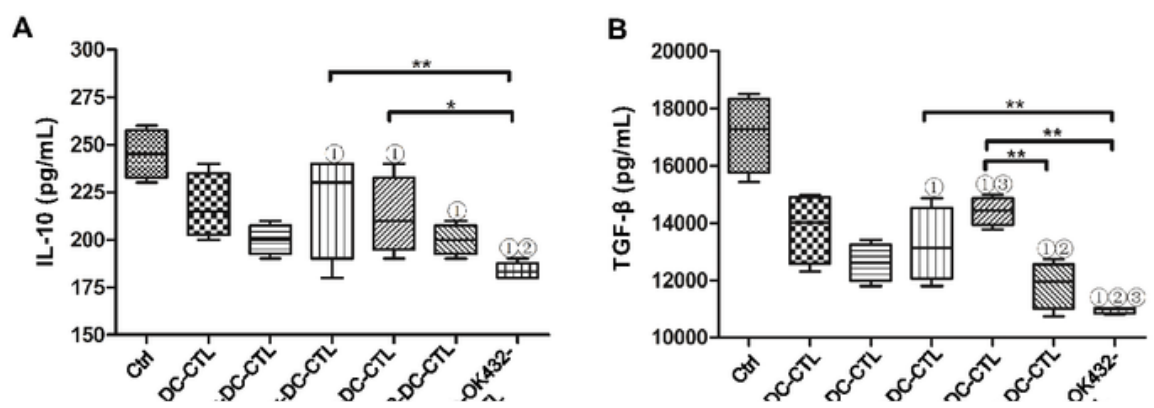

Figure 7

CD40 Ab and OK-432 synergistically enhance the secretion of stimulatory cytokines whereas inhibit the inhibitory cytokines. 
A. CTLs induced by CD40 Ab and OK-432 inhibit the production of IL-10. The inhibitory cytokine IL-10 is restrained by the agent-treated groups, and the combinational group shows a prominent suppression.

B. CTLs induced by CD40 Ab and OK-432 inhibit the production of TGF- $\beta$. The secretion of inhibitory cytokine TGF- $\beta$ is blocked by the agent-treated groups, and the combinational group contributes the significant change.

C. CTLs induced by CD40 Ab and OK-432 augment the generation of IL-12. Although the four agenttreated groups confer to the enhancement of stimulatory cytokine IL-12, the combinational group exhibits the outstanding function.

D. CTLs induced by CD40 Ab and OK-432 augment the secretion of IFN- - . The four agent-treated groups facilitate the increasing of stimulatory cytokine IFN- $\gamma$, and the combinational group displays an obvious advantage.

\section{Supplementary Files}

This is a list of supplementary files associated with this preprint. Click to download.

- Supplementalmaterial.docx 